\title{
The Effect of Demineralized Bone Matrix on the Healing of Intramembranous Bone Grafts in Rabbit Skull Defects
}

\author{
A.B.M. Rabie*, Y.M. Deng, N. Samman', and U. Hägg \\ Department of Children's Dentistry and Orthodontics, and ${ }^{1}$ Department of Oral and Maxillofacial Surgery, Faculty of Dentistry, The \\ University of Hong Kong, 34 Hospital Road, Hong Kong; ${ }^{*}$ to whom correspondence and reprint requests should be addressed
}

\begin{abstract}
A clinical dilemma exists regarding the type of bone that should be used to replace diseased or traumatized osseous tissue. Oral, plastic, and orthopedic surgeons normally implant viable mineralized endochondral (EC) autografts or demineralized EC allografts. A few clinicians have recognized the disadvantages of using EC bone in craniofacial surgery and advocated the replacement of intramembranous (IM) bone with healthy IM bone. However, controversy and uncertainty surround our understanding of these matrices to induce bone formation. Recent studies have advocated the use of other materials with osteoinductive properties, such as demineralized bone matrix (DBM). The proposed delivery system used in this study included IM bone grafts, DBM, and fixation of the IM bone graft. The purpose of this work was to gain further insights into the mechanism of healing of IM bone, in both the presence and the absence of DBM, and to compare the healing of IM bone grafts with that of DBM alone. Criticalsized $(10 \times 5 \mathrm{~mm})$, full-thickness bony defects in rabbit parietal bone, devoid of periosteum, were filled with IM bone graft (mandible) alone, demineralized cortical bone matrix (DBM) alone, or combined DBM-IM bone graft, or were left unfilled. Histologic changes were examined 14 days later. The IM bone graft healed through IM ossification with no intermediate cartilage stage. DBM and composite DBM-IM healed through an EC ossification with an intermediate cartilage stage. It is hypothesized that the role of the IM graft is to induce neovascularization into the defect site, and that the undifferentiated mesenchymal cells in the perivascular region of the new blood vessels are induced by the bone morphogenetic protein(s) in the DBM into bone-forming cells.
\end{abstract}

Key words: demineralized bone matrix, intramembranous bone graft, endochondral ossification, intramembranous ossification.

Received December 20, 1994; Accepted November 8, 1995

\section{Introduction}

Bone formation occurs by either endochondral (EC) or intramembranous (IM) ossification. In EC ossification, bone replaces a hyaline cartilage model. Long bones, such as the femur and humerus, form initially by this pathway (Mckibbin, 1978). In IM ossification, bone replaces stromal connective tissue proper, without the intermediate stage of cartilage formation. Both the frontal and parietal bones of the skull vault form via the IM ossification pattern (Fawcett, 1986).

At present, in the clinic, fresh autogenous EC or IM bone grafts are used to regenerate bone in osseous defects. However, controversy and uncertainty surround our understanding of their ability to induce bone formation. Previous work suggested that subcutaneous implants of both demineralized EC and IM bone matrices induce ossification through an intermediate cartilage formation stage (Reddi and Huggins, 1972). Recently, Scott and Hightower (1991) demonstrated that implants of demineralized EC bone matrix induced bone formation via a cartilage intermediate stage; however, in contrast to previous work (Reddi and Huggins, 1972; Isaksson and Alberius, 1992), they found that demineralized IM bone matrix induced bone without an intermediate cartilage stage and proceeded directly through an IM ossification pathway.

Clinical studies have successfully used demineralized bone matrix (DBM) as bone graft material for the treatment of acquired and congenital cranio-maxillofacial and orthopedic defects (Urist, 1976; Glowacki et al., 1981). This natural biomaterial is utilized in osseous reconstruction because of its ability to induce differentiation of mesenchymal cells within non-skeletal tissue into chondroblasts followed by cartilage and subsequent bone formation (Urist, 1965; Reddi and Huggins, 1972; Chalmers et al., 1975; Tuli and Singh, 1978). Recent clinical observations confirm some degree of success of composite bone grafting with demineralized bone matrix (DBM) (Johnson et al., 1988; Urist, 1991). Others (Mulliken and Glowacki, 1980; Sonis et al., 1983) have already used DBM and bone graft combinations clinically and suggested that bone formation induced by the DBM, in conjunction with a suitable delivery 
system, may be important in clinical situations requiring bone replacement and new bone formation. Clinically, autogenous IM bone grafts were used in conjunction with demineralized cortical bone matrix (DBM) for the repair of mandibular and maxillary defects in humans, while in the laboratory, the healing of surgically induced bony defects in rat mandibles, by means of IM bone grafts and DBM, was shown to progress through an intermediate cartilage stage (Rabie et al., 1994). It is hypothesized that the role of the IM graft is to induce neovascularization into the defect site, and that the undifferentiated mesenchymal cells in the perivascular region of the new blood vessels are induced by the bone morphogenetic protein(s) in the DBM into boneforming cells.

As noted, previous work (Mulliken and Glowacki, 1980; Sonis et al., 1983; Johnson et al., 1988; Urist, 1991; Rabie et al., 1994) demonstrated therapeutic potential for the use of composite bone grafting with demineralized bone matrix. However, the bone induction process in this delivery system remains unclear, and the understanding of the underlying bone induction mechanisms is incomplete. The objectives of the present study are to gain further insights into the mechanism of healing of IM bone graft both in the presence and in the absence of DBM, and to compare the healing of IM bone grafts with that of DBM alone. In this way, a sound foundation may be established for the clinical use of these procedures in the treatment of craniofacial bony defects in humans-for example, in the repair of alveolar clefts.

\section{Materials and methods}

\section{Experimental groups}

Twelve adult New Zealand White rabbits $(3.9 \pm 0.4 \mathrm{~kg})$ were used as the experimental model. Four groups, with three rabbits in each, were subjected to different treatments: (I) control (no graft material), (II) IM bone graft alone, (III) DBM alone, and (IV) combined DBM-IM bone graft.

\section{Surgical procedures}

A standardized surgical procedure was performed on all the animals. This protocol was reviewed and approved by the Hong Kong Government, Department of Health, Control of Animal Experiments and by the Committee on the Use of Live Animals for Teaching and Research, Hong Kong University. To control for variance in technique, the same surgeon performed all operations using identical bone-grafting harvest and inset techniques.

After administering pre-medication $(30 \mathrm{mg} / \mathrm{kg}$ Oxytetracycline hydrochlorine, and $50 \mathrm{\mu g} / \mathrm{kg}$ Temgesic) as a pre-operative antibiotic and analgesic, the surgeon anesthetized the rabbits by intramuscular (gluteal muscle) Hypnorm $(0.3$ $\mathrm{mL} / \mathrm{kg}$ ) and Valium $(1 \mathrm{mg} / \mathrm{kg}$ ), with systemic administration in increments of Hypnorm at $0.1 \mathrm{~mL} / \mathrm{kg}$ every $30 \mathrm{~min}$.

Two $10 \times 5 \mathrm{~mm}$ full-thickness bone defects, devoid of periosteum, were produced in both sides of the parietal bone region by means of a low-speed dental drill and bur. The mandible was used as the donor site for the two $10 \times 5 \mathrm{~mm}$ IM bone grafts. Both upper and lower sites of the bone graft and parietal bone
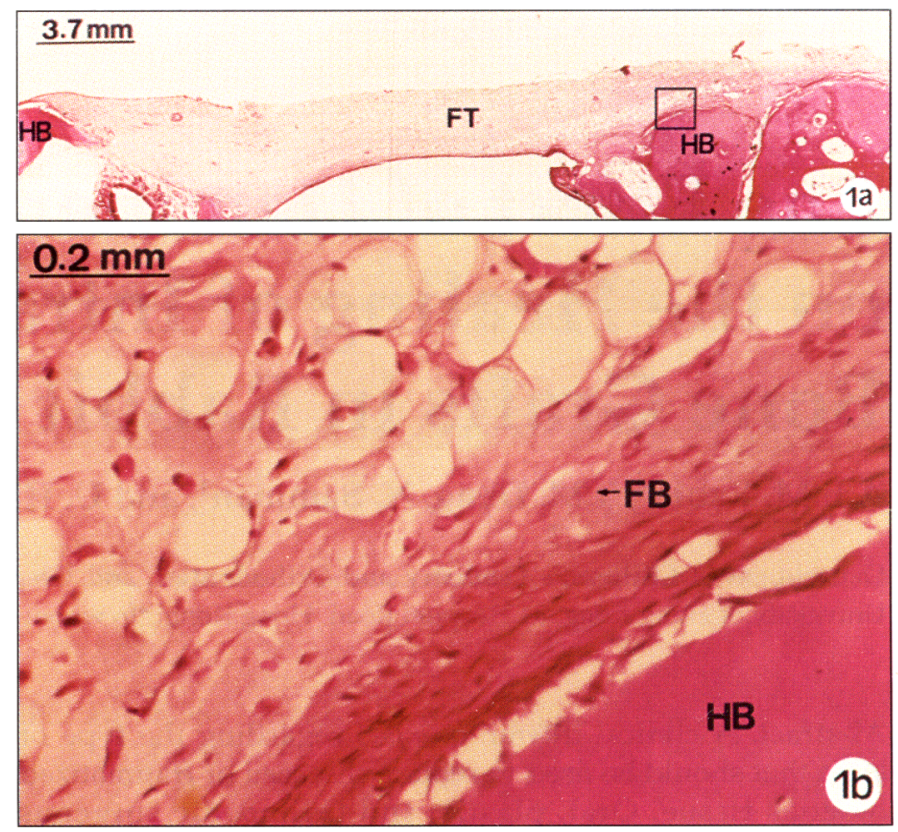

Figure 1. Histological evaluation of control defect (stained with HPS). (a) The defect is filled with fibrous connective tissue (FT), and host bone is marked as HB. 5x. (b) Higher magnification showing fibrous connective tissue and fibroblasts (FB). 101x.

were punctured in two sites for fixation with $0.3-\mathrm{mm}$ ligature ties.

Group I, Control: The critical size defect was left without graft material.

Group II, IM bone graft alone: The IM bone graft was placed into the defect and fixed in place as described above.

Group III, DBM alone: The defects were packed with demineralized cortical bone powder (Musculoskeletal Transplant Foundation, Holmdel, NJ, USA) mixed with whole blood.

Group IV, combined DBM-IM bone graft: The IM bone graft was sandwiched between two layers of the demineralized cortical bone powder that was mixed with whole blood, and fixed in place as described above.

All wounds were closed with 4-0 nylon suture. No attempts were made to suture periosteum together. Post-operatively, the rabbits were given an antibiotic $30 \mathrm{mg} / \mathrm{kg}$ body weight, i.m. (Oxytetracycline Hydrochlorine), daily for 10 days, and for pain relief, Temgesic $50 \mu \mathrm{g} / \mathrm{kg} \mathrm{s} / \mathrm{c}$ daily for two weeks.

\section{Histology}

Two weeks post-surgically, the animals were killed with Pentobarbital Sodium, i.v., $60 \mathrm{mg} / \mathrm{kg}$ body weight, and the defect areas, including the surrounding tissue, were harvested for histological preparation. Tissues were fixed in 10\% neutral phosphate-buffered formalin, decalcified in K's Decal Fluid for 2 days, and double-embedded in Celloidin-Paraffin. The specimen was cut into serial coronal sections $5 \mathrm{~mm}$ thick and stained with hematoxylin-phloxine and saffron (HPS). So that pre-osteoblasts and osteoblasts could be identified, semi-thin sections stained with toluidine blue were also performed in the IM bone graft group. Staining for possible cartilage formation 

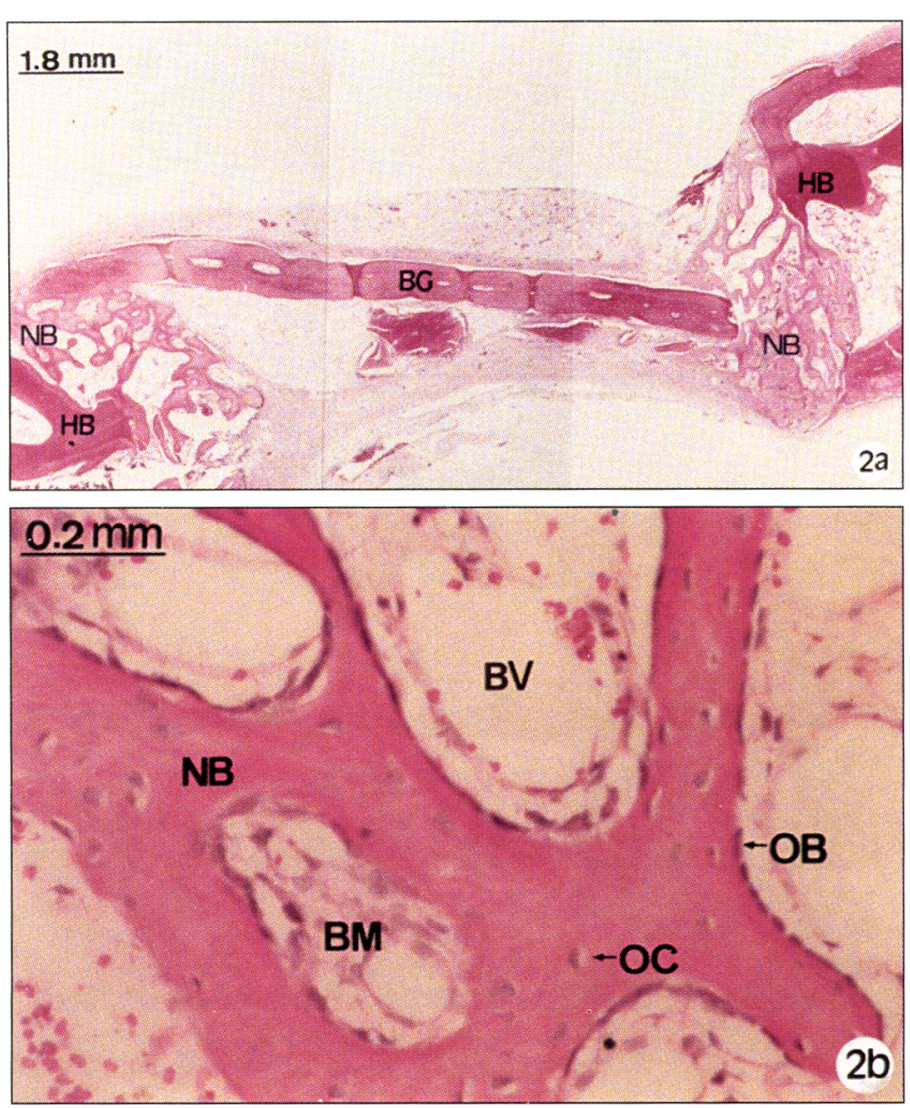

Figure 2. Histologic evaluation of defect filled with IM bone graft (stained with HPS). (a) The new bone (NB) is bridging from host bone (HB) to bone graft (BG). 11x. (b) The newly formed bone (NB) with embedded osteocytes (OC) is observed, and osteoblasts (OB) are present on the surface of the newly formed bone. Note that bone marrow elements are now present (BM). 101x.

was performed with alcian blue and van Giesen's staining. For identification of mesenchymal cells, the specimen was stained with anti-vimentin antibody. The specimens were qualitatively evaluated by means of an Olympus transmitting light microscope (BH-2, Tokyo, Japan).

\section{Results}

\section{Clinical and physical examination}

All animals remained in excellent health throughout the course of the experiment and rapidly recovered after surgery. There was no evidence of infection or other complications in any animal.

\section{Gross appearance}

Two weeks post-surgically, control (unfilled) defects did not exhibit bone formation, since they were filled with soft tissue. Defects implanted either with IM-Grafts, DBM, or DBM-IM grafts were grossly filled with hard tissue.

\section{Histologic findings}

Histologic examination confirmed the gross findings. The
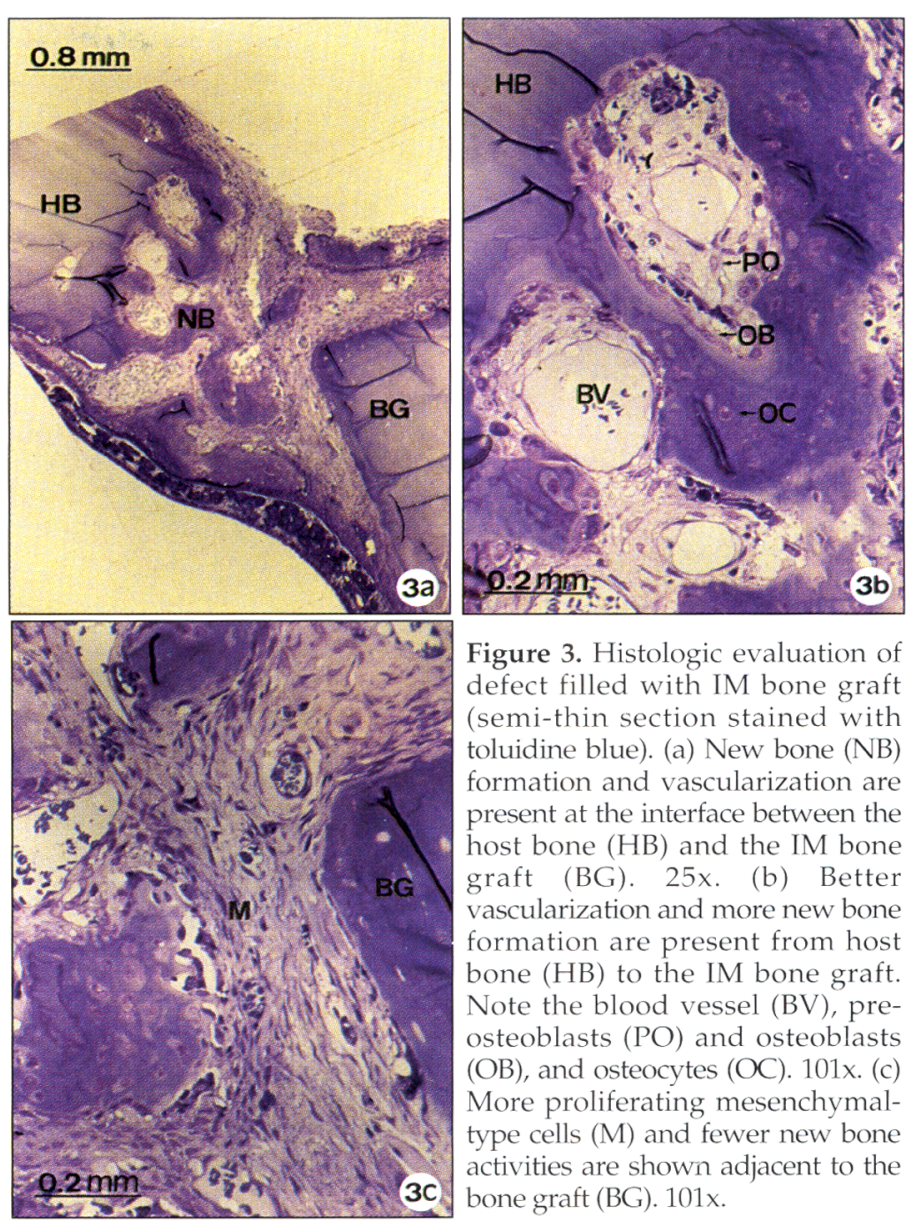

Figure 3. Histologic evaluation of defect filled with IM bone graft (semi-thin section stained with toluidine blue). (a) New bone (NB) formation and vascularization are present at the interface between the host bone (HB) and the IM bone graft (BG). 25x. (b) Better vascularization and more new bone formation are present from host bone $(\mathrm{HB})$ to the IM bone graft. Note the blood vessel (BV), preosteoblasts (PO) and osteoblasts $(\mathrm{OB})$, and osteocytes (OC). 101x. (c) More proliferating mesenchymaltype cells $(\mathrm{M})$ and fewer new bone activities are shown adjacent to the bone graft (BG). 101x.

control defects were filled by fibrous connective tissue (Figs. $1 \mathrm{a}, 1 \mathrm{~b})$, and, occasionally, a narrow margin of new bone formation could be seen around the edges of the defects.

The healing of the IM bone graft group showed a route of entry for osteoblasts and osteoclasts (Figs. 2-4). The newly formed bone with osteoblasts and osteocytes was observed within the interface from the host bed to the graft (Figs. 2a, $3 a)$, neovascularization (blood vessels) was present along the border of the host bone (Figs. 2b, 3b), and numerous mesenchymal cells were shown adjacent to the bone graft (Fig. 3c). Occasional osteoclasts were seen along the host bone. There appeared to be vascular proliferation and new bone formation from the host bone toward the graft bone. At 14 days, no hyaline cartilage was seen in the IM bone graft group (Figs. 4a, 4b).

The group treated with DBM was characterized by EC ossification concomitant with resorption of the original DBM (Fig. 5). Vascular invasion and new bone formation appeared at the host bone edges and progressed toward the middle of the defect (Fig. 5a). Chondrocytes with their typical territorial cartilage matrix were interposed between some of the DBM particles (Fig. 5b), and alcian blue staining showed positive results. In the middle of the defect, abundant proliferating mesenchymal cells, positively stained for vimentin, appeared between the particles of DBM (Figs. 5a, 5c). Inflammatory cells, polymorphonuclear leukocytes (PNL), could also be seen as a response to the surgically induced defect. 



Figure 4. Histologic evaluation of cartilage present in IM graft (alcian blue and van Geisen's staining). (a and b) New bone does not stain blue, showing a negative result, meaning that no hyaline cartilage is present. Instead, new bone formation and good vascularization are typically observed. The host bone is marked as HB. The same blood vessel (BV) and new bone formation (NB) are shown at low magnification $(a, 25 x)$ and at high magnification $(b, 101 x)$.

The group treated with combination DBM-IM graft material was also characterized by EC ossification (Fig. 6). Vascular invasion and new bone formation occurred throughout (Fig. 6a), unlike the IM graft alone, which was localized at the interface between the host bone edge and bone graft (Fig. 2). Islands of chondrocytes and cartilage matrix were seen between pieces of DBM and also adjacent to the bone grafts (Figs. 6b, 6c). Also, there was an abundance of proliferating mesenchymal type cells present in the middle of the defect.

\section{Discussion}

Repair of large bony defects in the craniofacial region represents a major challenge for the surgeon, since autogenous graft material may not be available in sufficient amounts. Recent studies have advocated the use of other materials with osteoinductive properties, such as demineralized bone matrix (Urist and Strates, 1970; Glowacki et al., 1981). A novel delivery system, including IM bone grafts, demineralized cortical bone matrix, and fixation of the IM bone graft, was used in this study (Rabie et al., 1994).
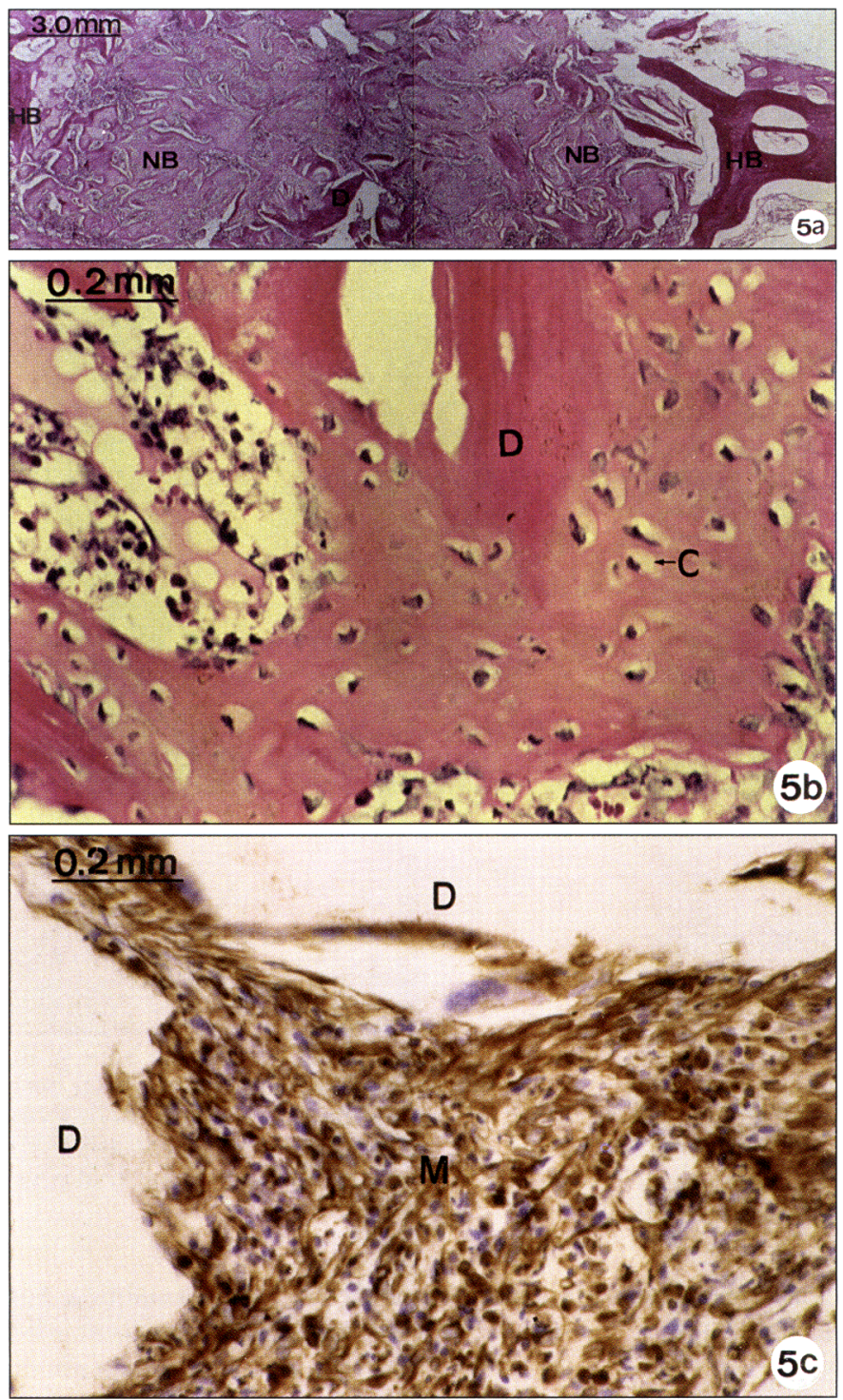

Figure 5. Histologic evaluation of defect filled with DBM. (a) New bone (NB) formation is bridging from the host bone (HB) to the middle of the defects (stained with HPS). 7x. (b) Note that a cartilage matrix with chondrocytes $(C)$ is present adjacent to the DBM (D) (stained with HPS). 101x. (c) In the middle of the defect, abundant proliferating mesenchymal cells (M), positively stained for vimentin, appeared between the particles of DBM (D) (stained with anti-vimentin antibody). 101x.

The rabbit model used in this study was relevant because the non-grafted control defect did not heal. The area was large $(10 \mathrm{~mm} \times 5 \mathrm{~mm}$ is a significant defect for the parietal bone of a rabbit) and of full thickness, and the periosteum was totally disrupted and not replaced, as shown in the control group. Therefore, in this model, healing must be largely attributed to the implant material.

Demineralized bone matrix induces new bone formation in a multi-step cascade (Urist et al., 1982; Vandersteenhoven and Spector, 1983; Reddi et al., 1987). The major phases of osteoinduction are chemotaxis, mitosis of mesenchymal cells by day 3, differentiation of host mesenchymal cells to chondroblasts by day 5, and numerous chondrocytes 

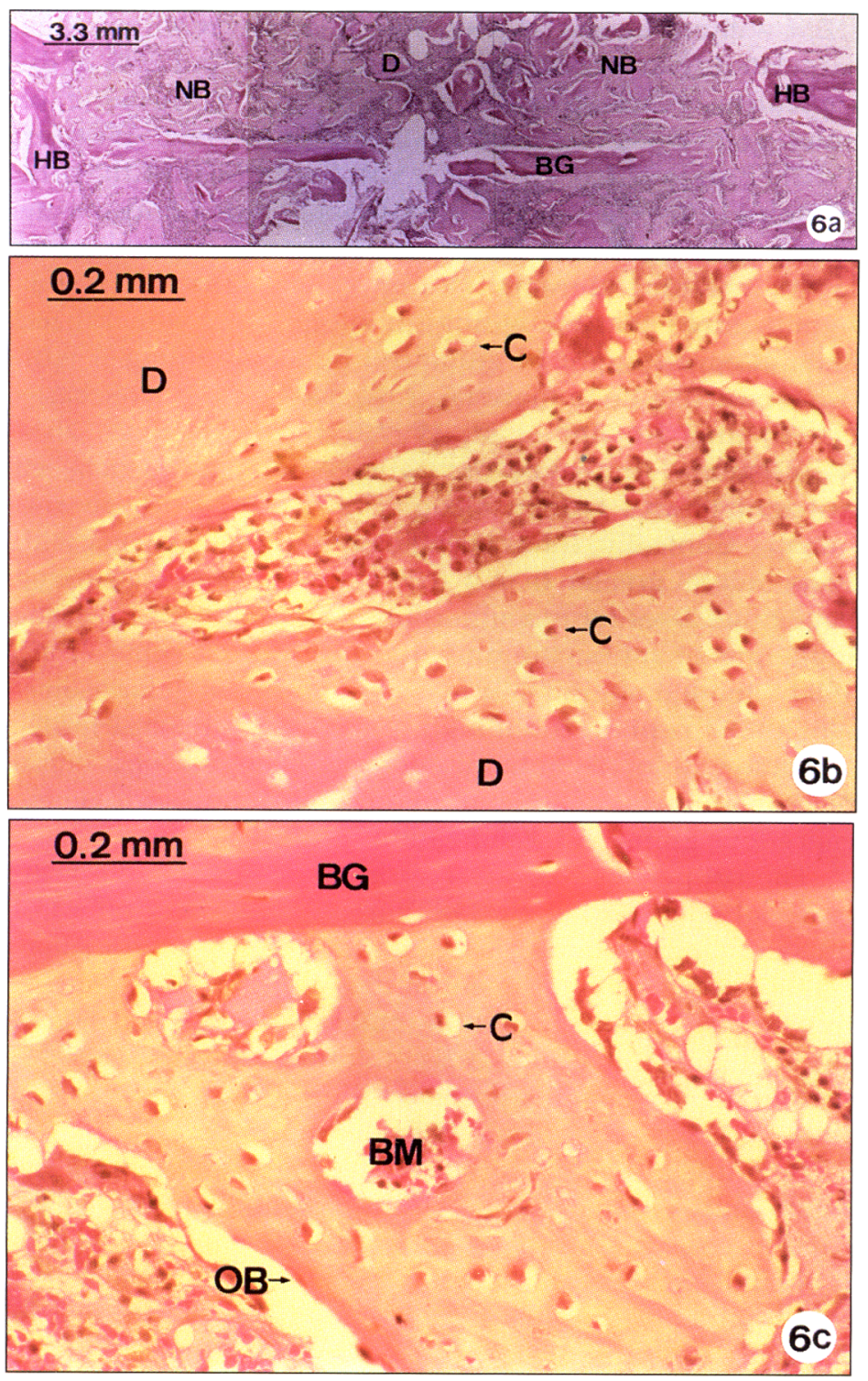

Figure 6. Histologic evaluation of defect filled with DBM-IM graft (stained with HPS). (a) New bone formation (NB) is bridging from the host bone (HB) to the middle of the defects. IM bone graft is marked as BG. 6x. (b) Cartilage matrix along with chondrocytes (C) is interposed between the DBM particles (D). 101x. (c) Cartilage matrix with chondrocytes $(\mathrm{C})$ covered by a layer of osteoblasts $(\mathrm{OB})$ are forming adjacent to the bone graft (BG). Note the location of the bone marrow space (BM). 101x.

present on days 7 to 8 . With the advent of capillary invasion on day 9, chondrocytes showed signs of hypertrophy, and the first signs of mineral formation are evident in the matrix of the hypertrophic chondrocyte. By days 10 to 11, numerous multinucleated chondroblasts appear close to the regions of chondrolysis. Concurrently, new bone is formed on the surface of calcified matrix and the implanted matrix. Multinucleated osteoclasts remodel the newly formed bone by days 12 to 18 , resulting in selective dissolution of the implanted matrix. At this stage, some areas in the defect contain bone and others still contain cartilage. Thus, in this study, we chose to evaluate the healing pattern of composite bone grafts and compare it with that of the DBM alone in 14 days. This allows both cartilage and bone to co-exist in the same section (Figs. 5b, 6b, 6c).

A significant finding in this study is that IM bone grafts induced bone formation through IM ossification (Figs. 2-4). The present results support the view that IM bone, when used as a graft, has osteogenic potential (Ray and Sabet, 1963). To date, almost all known bone-derived osteoinductive factors have been isolated from EC bones and initiate bone induction via EC ossification. Recently, a heparin-sepharose-binding, osteoinductive factor was extracted and partially purified from bovine IM bone matrix, and exhibited a molecular weight different from that of a comparable osteoinductive/condroinductive factor isolated from EC bone (Scott et al., 1994). Their work is the first demonstration that osteoinductive factors may also be present in osseous tissue which forms initially and completely via IM ossification, and points to a new source of osseous tissue from which such factors can be isolated. These results are valuable in clinical settings where it would be more appropriate to replace traumatized or diseased IM bone with IM rather than with EC bone (Scott and Hightower, 1991).

Histologic analysis demonstrated that IM bone grafts induced bone formation via IM ossification, in contrast to implants of DBM, which induced bone via EC ossification. Although Reddi and Huggins (1972) suggested that IM matrix also induces the EC cascade, the present results support the findings of Scott and Hightower (1991), which also compared the bone-inductive capacities of EC and IM demineralized bone matrices. Morphological and radiolabeling techniques demonstrated that implants of EC bone matrix induced bone formation via EC ossification. In contrast, implants of IM bone matrix induced bone only through IM ossification. Our findings support these latter observations (Figs. 2-4). Some early work by Pritchard (1946) observed that, in 22 of 23 fractures of the parietal bones of rats, healing proceeded with no cartilage intermediate stages. These findings suggest that the matrix of IM bone differs qualitatively from that of EC bone in terms of their respective abilities to induce cartilage and/or ultimately bone formation.

The most interesting finding in this study was that combining DBM with IM bone altered the healing of IM bone to an EC ossification instead of osteogenic ossification route. The hypothesis of this study entertained the possibility that the IM autogenous grafts induce neovascularization into the defect area, bringing undifferentiated mesenchymal cells, which could then be acted upon by the DBM to induce their differentiation into chondroblasts. IM autogenous grafts may contribute to angiogenesis and increase bone ingrowth from host bone margins. IM grafts may be calibrated to deploy factors at the proper location and time, and ultimately biodegrade to biocompatible products as the new bone is regenerated. Also, IM bone graft may function as a temporary stabilizer until viable osseous continuity is obtained as a primer for new bone formation, and it may act as a mechanical barrier to fibroblasts covering the dura. Fibroblasts may interfere with the process of osteogenesis. However, results of the present study could not directly support all of these speculations. Further biochemical as well as quantitative analysis will focus on the utility of the 
composite DBM-IM group both experimentally and clinically. Furthermore, the fact that the method of fixation used in this study was not rigid fixation could have affected the healing of the IM group to adopt an endochondral ossification route. However, healing of the IM group showed an osteogenic route, while that of the DBM-IM group showed an EC ossification route. Since the healing of the DBM-alone group showed a cartilage intermediate, then the endochondral ossification in the DBM-IM group is more likely to be due to the effect of the DBM and not due to the non-rigid fixation. It is well-known that DBM induces new bone formation (Urist et al., 1967). Urist and others demonstrated this potent osteogenic promoter in the reconstruction of skeletal defects (Urist, 1976; Tuli and Singh, 1978; Takagi and Urist, 1982; Wittbjer, 1983). However, DBM was not used in combination with IM autogenous bone grafts. The advantage of DBM is thought to be due to the content and diffusibility of activated graft bone-bound TGF$\beta$ (a member of the bone morphogenetic protein family) and other growth factors that interact with undifferentiated osteogenic precursor cells in the host bed (Wozney et al., 1988; Urist, 1989), thus causing them to proliferate and differentiate into functional chondrocytes and osteoblasts (Harakas, 1984; Wozney et al., 1988; Urist, 1989). Conceptually, then, such IM graft-DBM composites should retain more normal biomechanical properties; however, the literature provides no clues as to the healing of such composite grafts. In the present study, the histologic analysis of the DBM-IM grafted implants showed that the healing was characterized by EC ossification (Fig. 6). This suggested that DBM could be a useful supplementary osteoinductivecompetent material. Since DBM could not diffuse into the IM bone when mixed with it, cartilege never developed within the IM-grafted vascular canals.

The question remains why the DBM-IM-grafted implants healed through EC ossification. It is probably because the resident mesenchymal cells have osteogenetic determination. Osteogenetic competence or determination is a state of readiness but not yet activated capacity of mesenchymal cells to differentiate into bone cells. The basis of osteogenetic competence is a group of gene-action systems for production of cartilage, bone, and bone marrow cells. Determination of bone cell specialization is therefore a heritable or genetic property (Urist and Strates, 1971). It should be stated that the DBM-IM composite-material consistently facilitated the thickest, most stable, and optimal contour-preserving bone tissue repair.

The significance of these results is best appreciated when considered in light of recent advances in osseous repair. The osteoinductive proteins, bone morphogenetic protein and osteogenin, have been shown to induce complete osseous repair in similar defects. Germane to the present results are the indications from these and other studies that have proven that the clinical effectiveness of osteoinduced bony repair requires some type of existing defect-filling material. Not only does this filling material prevent tissue collapse into the defect during the healing phase, but it also acts as a scaffolding and potential carrier vehicle.

Reconstruction of large skeletal defects by implanting
DBM without a skeletal substitute has been reported to be associated with incomplete bridging in $19 \%$ and $25 \%$ of the implants, respectively, where either allogeneic or autologous DBM was used (Tuli and Singh, 1978; Wittbjer, 1983). Previous experimental studies indicated that the combination of a bone-inductive substance and a nonviable bone graft may be an advantage for reconstructing large skeletal defects (Yang et al., 1994). It was hypothesized that the biological success of a mineralized bone allograft might be enhanced by surrounding it with osteoinductive bone material. Also, Köhler and Kreicbergs (1987) and Köhler et al. (1990) reported that DBM supplements improved the osteogenicity of autoclaved autografts and frozen allografts in rabbit ulnae and humeri. Clinically, it has been suggested that patients with bone defects greater than 4 to $6 \mathrm{~cm}$ have been considered poor candidates for cancellous bone grafting because of the high rate of refracture and non-union (Enneking et al., 1980; Moore et al., 1983; Goldströhm et al., 1984). Johnson et al. (1988) reported that six patients with traumatic segmental 3- to 17-cm tibial defects developed solid union by implantation of (recombinant) hBMP and autogeneic cancellous grafts and stabilization.

The properties that are required for an effective graft material (Gepstein et al., 1987) are: (1) the recruitment of osteogenetic precursor cells to the site of new-bone formation, (2) the support of attachment of these cells to a substrate so that they can proliferate, (3) the induction of differentiation of the resident stromal cells to form bone, and (4) neovascularization for support of the newly formed bone. The present proposed delivery system has these characteristics.

Because of the factors present in IM bone, and the osteoinductive factors in DBM, this system, if confirmed by further experimentation, would be of critical value to clinicians who intend to use this system to repair nonregenerative bony defects. Future studies are indicated to determine the long-term efficacy of this delivery system, and we will continue to explore and investigate the fundamental biochemical principles governing these complex processes.

\section{Acknowledgments}

This work was supported by CRC Grants 372/251/6435 and $335 / 253 / 0012$, The University of Hong Kong. The skillful technical assistance of Mr. Michael Pang, Mr. Man Tung Lee, and Mr. K.Y. To is gratefully acknowledged. This study was partially supported by an IADR Norton M. Ross Fellowship awarded to Dr. Deng (School of Stomatology, Beijing Medical University). The fellowship period was spent at the Division of Oral Biology, Northwestern University, under the supervision of Dr. Michael T. DiMuzio.

\section{References}

Chalmers J, Gray DH, Rush J (1975). Observations on the induction of bone in soft tissues. J Bone Joint Surg 57(B):36-45.

Enneking WF, Eady JL, Burchardt H (1980). Autogenous cortical bone grafts in the reconstruction of segmental skeletal defects. J Bone Joint Surg 62(A):1039-1058. 
Fawcett DW (1986). Bone: Histogenesis of bone. In: A textbook of histology. 7th ed. Philadelphia: Saunders, pp. 216-221.

Gepstein R, Weiss RE, Hallel T (1987). Bridging large defects in bone by demineralized bone matrix in the form of a powder. A radiographic, histological, and radioisotopeuptake study in rats. J Bone Joint Surg 69A:984-992.

Glowacki J, Kaban LB, Murray JE, Folkman J, Mulliken JB (1981). Application of the biological principle of induced osteogenesis for craniofacial defects. Lancet 1:959-962.

Goldströhm GL, Mears DC, Swartz WM (1984). The results of 39 fractures complicated by major segmental bone loss and/or leg length discrepancy. J Trauma 24:50-58.

Harakas NK (1984). Demineralized bone-matrix-induced osteogenesis. Clin Orthop 188:239-251.

Isaksson S, Alberius P (1992). Comparison of regenerative capacity elicited by demineralized bone matrix of different embryonic origins. J Craniomaxillofac Surg 20:73-80.

Johnson EE, Urist MR, Finerman GAM (1988). Repair of segmental defects of the tibia with cancellous bone grafts augmented with human bone morphogenetic protein. A preliminary report. Clin Orthop 236:249-257.

Köhler P, Kreicbergs A (1987). Incorporation of autoclaved autogeneic bone supplemented with allogeneic demineralized bone matrix: An experimental study in the rabbit. Clin Orthop 218:247-258.

Köhler P, Ehrnberg A, Kreicbergs A (1990). Osteogenic enhancement of diaphyseal reconstruction. Acta Orthop Scand 61:42-45.

Mckibbin B (1978). The biology of fracture healing in long bones. J Bone Joint Surg 60(B):150-162.

Moore JR, Weiland AJ, Daniel RK (1983). Use of free vascularized bone grafts in the treatment of bone tumors. Clin Orthop 175:37-44.

Mulliken JB, Glowacki J (1980). Induced osteogenesis for repair and construction in the craniofacial region. Plast Reconstr Surg 65:553-560.

Pritchard JJ (1946). Repair of fractures of the parietal bone in rats. J Anat 80:55-62.

Rabie AM, Lonergan M, Forbes D (1994). An experimental study of the effects of fixation versus non-fixation on the healing of onlay bone grafts (abstract). Eur J Orthod 16:347.

Ray RD, Sabet TY (1963). Bone grafts: Cellular survival versus induction. An experimental study in mice (abstract). J Bone Joint Surg 45(A):337.

Reddi AH, Huggins C (1972). Biochemical sequences in the transformation of normal fibroblasts in adolescent rats. Proc Natl Acad Sci USA 69:1601-1605.

Reddi AH, Weintraub S, Muthumaran N (1987). Biologic principles of bone induction. Orthop Clin North Am 18:207-212.
Scott CK, Hightower JA (1991). The matrix of endochondral bone differs from the matrix of intramembranous bone. Calcif Tissue Int 49:349-354.

Scott CK, Bain SD, Hightower JA (1994). Intramembranous bone matrix is osteoinductive. Anat Rec 238:23-30.

Sonis ST, Kaban LB, Glowacki J (1983). Clinical trial of demineralized bone powder in the treatment of periodontal defects. J Oral Med 38:117-122.

Takagi K, Urist MR (1982). The role of bone marrow in bone morphogenetic protein-induced repair of femoral massive diaphyseal defects. Clin Orthop 171:224-231.

Tuli SM, Singh AD (1978). The osteoinductive property of decalcified bone matrix. J Bone Joint Surg 60(B):116-122.

Urist MR (1965). Bone formation by autoinduction. Science 150:893-899.

Urist MR (1976). Practical application of basic research on bone graft physiology. Instructional course lectures. Am Acad Orthop Surg Course Lectures 1-26.

Urist MR (1989). Bone morphogenetic protein, bone regeneration, heterotopic ossification and the bone-bone marrow consortium. In: Bone and mineral research. Vol. 6. Peck WA, editor. Amsterdam: Elsevier Science, p. 57.

Urist MR (1991). Emerging concepts of bone morphogenetic protein. In: Fundamentals of bone growth: Methodology and applications. Dixon AD, Sarnat BG, Hoyte DAN, editors. Boston: CRC Press, pp. 189-198.

Urist MR, Strates BS (1970). Bone formation in implants of partially and wholly demineralized bone matrix. Clin Orthop 71:271-278.

Urist MR, Strates BS (1971). Bone morphogenetic protein. J Dent Res 50(Suppl 6):1392-1406.

Urist MR, Silverman BF, Büring K, Dubuc FL, Rosenberg JM (1967). The bone induction principle. Clin Orthop 53:243-283.

Urist MR, Lietze A, Mizutani H, Takagi K, Triffitt JT, Amstutz J, et al. (1982). A bovine low molecular weight bone morphogenetic protein (BMP) fraction. Clin Orthop 162:219-232.

Vandersteenhoven JJ, Spector M (1983). Histological investigation of bone induction by demineralized allogeneic bone matrix: A natural biomaterial for osseous reconstruction. J Biomed Mater Res 17:1003-1014.

Wittbjer J (1983). Bone matrix and bone formation. An experimental study of demineralized bone reimplanted in bone defects (thesis). Malmö, Sweden: University of Lund.

Wozney JM, Rosen V, Celeste AJ, Mitsock LM, Whitters MJ, Kriz RW, et al. (1988). Novel regulators of bone formation, molecular clones and activities. Science 242:1528-1534.

Yang CY, Simmons DJ, Lozano R (1994). The healing of grafts combining freeze-dried and demineralized allogeneic bone in rabbits. Clin Orthop 298:286-295. 